OPEN ACCESS

Edited by: Isabel Merida,

Spanish National Research Council, Spain

Reviewed by:

Narayanan Parameswaran, Michigan State University, USA

Kaoru Goto,

Yamagata University School of

Medicine, Japan

*Correspondence:

Taku Kambayashi

taku.kambayashi@uphs.upenn.edu

Specialty section:

This article was submitted to

Signaling,

a section of the journal

Frontiers in Cell and Developmental

Biology

Received: 01 July 2016

Accepted: 22 August 2016

Published: 07 September 2016

Citation:

Singh BK and Kambayashi T (2016) The Immunomodulatory Functions of Diacylglycerol Kinase $\zeta$.

Front. Cell Dev. Biol. 4:96.

doi: 10.3389/fcell.2016.00096

\section{The Immunomodulatory Functions of Diacylglycerol Kinase $\zeta$}

\author{
Brenal K. Singh and Taku Kambayashi * \\ Department of Pathology and Laboratory Medicine, Perelman School of Medicine at the University of Pennsylvania, \\ Philadelphia, PA, USA
}

The generation of diacylglycerol (DAG) is critical for promoting immune cell activation, regulation, and function. Diacylglycerol kinase $\zeta(D G K \zeta)$ serves as an important negative regulator of DAG by enzymatically converting DAG into phosphatidic acid (PA) to shut down DAG-mediated signaling. Consequently, the loss of DGK $\zeta$ increases DAG levels and the duration of DAG-mediated signaling. However, while the enhancement of DAG signaling is thought to augment immune cell function, the loss of DGK $\zeta$ can result in both immunoactivation and immunomodulation depending on the cell type and function. In this review, we discuss how different immune cell functions can be selectively modulated by DGK $\zeta$. Furthermore, we consider how targeting DGK $\zeta$ can be potentially beneficial for the resolution of human diseases by either promoting immune responses important for protection against infection or cancer or dampening immune responses in immunopathologic conditions such as allergy and septic shock.

Keywords: diacylglycerol, diacylglycerol kinase, signal transduction, immunomodulation, phospholipase C, extracellular signal-regulated kinase, phosphatidic acid

\section{INTRODUCTION}

Diacylglycerol (DAG) is a key secondary lipid messenger for transducing signals downstream of many receptors expressed by hematopoietic cells. DAG has shown to be important in driving the activation, proliferation, migration, and effector function of adaptive and innate immune cells. The generation of DAG can be accomplished by the activation of various cell-surface receptors, including $\mathrm{G}_{\mathrm{q}}$-mediated G-protein coupled receptors (GPCR)s (e.g., muscarinic and histamine receptors) and immunoreceptor tyrosine-based activation motif (ITAM)-bearing receptors [e.g., $\mathrm{T}$ cell receptor (TCR), FceRI] (Topham and Prescott, 1999; Kambayashi and Koretzky, 2007; SmithGarvin et al., 2009; Wright et al., 2013). The activation of these receptors results in the formation of proximal signaling complexes that are critical for the activation of phospholipase C (PLC). PLC activation leads to enzymatic cleavage of phosphoinositol 4,5-bisphosphate $\left(\mathrm{PIP}_{2}\right)$ into DAG and inositol 1,4,5-triphosphate $\left(\mathrm{IP}_{3}\right)$ (Imboden and Stobo, 1985). While $\mathrm{IP}_{3}$ mobilizes $\mathrm{Ca}^{2+}, \mathrm{DAG}$ activates the NF- $\mathrm{BB}$ and extracellular regulated kinase (ERK) pathways through protein kinase $\mathrm{C}$ (PKC) and RasGRP, respectively, to promote cell function (Tognon et al., 1998; Coudronniere et al., 2000; Dower et al., 2000; Sun et al., 2000; Wang et al., 2004; Quann et al., 2011). Consequently, the levels of DAG must be tightly regulated to control the magnitude and duration of the responses generated.

Diacylglycerol kinases (DGK) regulate DAG signaling by phosphorylating DAG and converting it into phosphatidic acid (PA) (Topham and Prescott, 1999; Joshi and Koretzky, 2013). The loss of DGKs increases DAG levels and the duration of DAG-mediated signaling. One might expect that elevated DAG levels would lead to general enhancement of effector responses. However, the 
enhancement of DAG signaling through the loss of DGKs can lead to either hyperactivation or hyporesponsiveness depending on the cell type and the type of response. There are 10 different isoforms comprising five different classes of DGKs, each of which control different cellular functions based on their distinct structural motifs and subcellular localization (Bunting et al., 1996; Goto and Kondo, 1996; Topham and Prescott, 1999; Kobayashi et al., 2007; Joshi and Koretzky, 2013). The three major isoforms that are abundantly expressed in lymphoid tissues are DGK $\alpha, \mathrm{DGK} \delta$, and DGK $\zeta$ (Shulga et al., 2011). In particular, mice that lack the zeta $(\zeta)$ isoform of DGK, which is highly expressed in hematopoietic cells, display profound effects on the functional behavior of various cell types. In the present review, we will focus on how DGK $\zeta$ plays both negative and positive roles in immune responses mounted by different cell types.

\section{NEGATIVE REGULATION OF EFFECTOR RESPONSES BY DGK $\zeta$ $\mathrm{CD}^{+}$and $\mathrm{CD}^{+}{ }^{+}$Conventional T Cells}

DGK $\zeta$ serves as a critical negative regulator of DAG signaling downstream of the TCR and can modulate the strength of TCR signaling. Early experiments using the immortalized Jurkat $\mathrm{T}$ cell line showed that overexpression of DGK $\zeta$ inhibits TCR signaling by reducing the levels of active GTP-bound Ras and, consequently, diminishing ERK activation (Zhong et al., 2002). Furthermore, the overexpression of DGK $\zeta$ was associated with decreased AP-1 transcription factor activity and CD69 expression (an early $\mathrm{T}$ cell activation marker) following TCR stimulation, both of which are regulated by the RasERK pathway. Importantly, $\mathrm{Ca}^{2+}$ flux was normal regardless of DGK $\zeta$ overexpression, suggesting that DGK $\zeta$ selectively regulated DAG-mediated signaling pathways downstream of the TCR. Further biochemical analysis through the use of a kinase dead DGK $\zeta$ mutant revealed that the enzymatic activity of DGK $\zeta$ was critical for its inhibitory effects on TCR signaling.

To test the physiological role of DGK $\zeta$ in T cells, Zhong et al. generated DGK $\zeta$ knockout mice (Zhong et al., 2003). Initial phenotypic analysis showed that DGK $\zeta$ KO mice contained similar frequencies and numbers of $\mathrm{CD} 4^{+}$and $\mathrm{CD} 8^{+} \mathrm{T}$ cells in secondary lymphoid organs and displayed no obvious defects in lymphoid architecture or cellularity. Furthermore, thymic development as analyzed by the number and frequency of CD4 single-positive (SP), CD8 SP, double-positive (DP), and double-negative $(\mathrm{DN})$ thymocytes in DGK $\zeta$-deficient mice was similar to wild-type (WT) mice. However, upon TCR stimulation, naïve DGK $\zeta \mathrm{KO} \mathrm{CD}^{+}$and $\mathrm{CD}^{+}{ }^{+} \mathrm{T}$ cells displayed enhanced upregulation of activation markers CD25 and CD69 and increased proliferation compared to WT T cells. The increased expression of activation markers was associated with enhanced phosphorylation of ERK but normal induction of non-DAG mediated pathways including $\mathrm{Ca}^{2+}$ flux and JNK activation. Importantly, bypassing TCR activation with a DAG analog, phorbol-12-myristate-13-acetate (PMA), abolished differences in activation between DGK $\zeta$ KO and WT T cells, suggesting that the hyperactivation of DGK $\zeta$ KO T cells was secondary to defective regulation of DAG.

In agreement with enhanced TCR signaling, DGK $\zeta$ KO $\mathrm{T}$ cells also display improved anti-viral responses (Zhong et al., 2003). DGK $\zeta$ KO mice infected with LCMV Armstrong showed enhanced viral-specific $\mathrm{T}$ cell responses as evidenced by decreased viral titers at day 7 following infection. This effect correlated with an increased number of total and $\mathrm{CD} 44^{\mathrm{hi}} \mathrm{CD} 62 \mathrm{~L}^{\text {lo }}$ effector $\mathrm{CD}^{+} \mathrm{T}$ cells in the spleen. Furthermore, LCMV-infected DGK $\zeta$ KO mice exhibited increased number of IFN $\gamma$-producing $\mathrm{CD}^{+}$and $\mathrm{CD} 4^{+} \mathrm{T}$ cells when restimulated with $\mathrm{LCMV}$-specific peptides, suggesting that DGK $\zeta \mathrm{KO} T$ cells displayed enhanced effector function following LCMV infection.

Similarly, DGK $\zeta$-deficient mice also exhibit enhanced antitumor responses. DGK $\zeta$ KO mice subcutaneously injected with OVA-expressing EL4 T cell lymphoma, had significantly reduced tumor mass compared to their WT counterparts (Riese et al., 2011). This effect was accompanied by an increased number of total and antigen-specific tumor-infiltrating $\mathrm{CD} 44^{\mathrm{hi}} \mathrm{CD}^{+} \mathrm{T}$ cells. To show that $\mathrm{T}$ cells were responsible for the enhanced anti-tumor effect by DGK $\zeta$ deficiency, DGK $\zeta$ KO and WT OVA-specific OT-I T cells were adoptively transferred into naïve recipient WT mice. Mice receiving DGK $\zeta$ KO compared to WT OVA-specific OT-I T cells also exhibited lower tumor mass upon challenge with OVA-expressing EL4 cells. Isolation of tumor-infiltrating OT-I cells revealed that the loss of DGK $\zeta$ increased the frequency of CD44 $4^{\mathrm{hi}}$ and IL-2-producing OT-I cells in a cell-intrinsic manner. In addition to preventing tumor engraftment, DGK $\zeta$ deficiency also improves tumor rejection of established tumors, as the adoptive transfer of DGK $\zeta$ KO but not WT OT-I effector T cells into tumor-bearing mice significantly reduced tumor burden (Riese et al., 2013). Thus, DGK $\zeta$ could represent a novel target for enhancing anti-tumor responses in adoptive immunotherapy. This could also be applied to engineered $\mathrm{T}$ cells that express chimeric antigen receptors (CAR) directed against the tumor, as DGK $\zeta$ deficiency was also shown to promote CAR T cell-mediated anti-tumor responses (Riese et al., 2013). How DGK $\zeta$ deficiency augments anti-tumor responses is unclear. Although DGK $\zeta \mathrm{KO} \mathrm{CD}^{+} \mathrm{T}$ cells display increased cytokine production and increased proliferation, their cytotoxic function is comparable to $\mathrm{WT} \mathrm{CD}^{+}{ }^{+} \mathrm{T}$ cells (Riese et al., 2011). Nevertheless, these studies demonstrate that DGK $\zeta$ serves to constrain $\mathrm{T}$ cell activation and anti-viral and anti-tumor $\mathrm{T}$ cell responses. Thus, inhibition of DGK $\zeta$ might provide a therapeutic opportunity to enhance immune-mediated viral and tumor clearance.

It is possible that DGK $\zeta$ is physiologically important for limiting over-activation and inducing anergy in inappropriately activated $\mathrm{T}$ cells. The expression level of DGK $\zeta$ can be controlled depending on the type of stimulation the $\mathrm{T}$ cell receives. $T$ cells that are stimulated through their TCR and co-stimulatory molecules downregulate DGK $\zeta$ transcript levels, thereby allowing appropriately activated $\mathrm{T}$ cells to become fully activated (Macian et al., 2002; Olenchock et al., 2006a; Zha et al., 2006). In contrast, $\mathrm{T}$ cells that receive TCR stimulation alone in the absence of co-stimulation do not downregulate DGK $\zeta$ levels, potentially leading to attenuated DAG-mediated signaling and 
decreased activation. Consistent with this notion, DGK $\zeta$ KO T cells resist anergy induction when activated by TCR alone in the absence of co-stimulatory signals (Olenchock et al., 2006a). In addition to TCR-mediated regulation, DGK $\zeta$ might also be regulated by environmental cytokines. In particular, IL-33 has been shown to up-regulate DGK $\zeta$ in cardiomyocytes following stimulation (Rui et al., 2012). Although it is unknown if IL-33 can upregulate DGK $\zeta$ in immune cells, it is tantalizing to speculate that cytokine signaling can affect the TCR responsiveness of T cells by regulating $\mathrm{DGK} \zeta$ levels.

\section{NK Cells}

NK cells are cytotoxic members of the innate lymphoid cell (ILC) family and play an important role in protection against viral infection and clearance of tumors (Artis and Spits, 2015). Unlike their adaptive counterparts $\left(\mathrm{CD}^{+} \mathrm{T}\right.$ cells), they do not possess a somatically-rearranged antigen receptor but rather express a variety of activating receptors specific for ligands displayed on virally-infected, stressed, or transformed cells (Lanier, 2008). NK cell activating receptors can be categorized into three main families based on the signaling adaptors used to relay downstream activation signals. These families include SAPdependent (e.g., 2B4), ITAM-dependent (e.g., CD16), or DAP10dependent (e.g., NKG2D) receptors (McVicar et al., 1998; Wu et al., 1999; Chen et al., 2006; Lanier, 2008). The activation of any of these three families of receptors relies on proximal signaling complexes involving SLP-76, which subsequently leads to the activation of PLC $\gamma$ and the production of DAG (Wu and Koretzky, 2004; Tassi et al., 2005; May et al., 2013). In addition to these activating receptors, NK cells express an assortment of inhibitory receptors, many of which bind to MHC class I alleles and negatively regulate activating receptor signaling by the recruitment of phosphatases such as SHP-1 and SHIP (Binstadt et al., 1996; Lanier, 2008).

NK cell activation is determined by the net balance of the activating and inhibitory inputs that the NK cell receives through its receptors. For example, NK cells are activated when neoplastic cells upregulate ligands such as RAE-1 or MICA, which are recognized by the activating receptor NKG2D (Jung et al., 2012). Likewise, NK cells are activated through disinhibition when tumor cells lose MHC class I, a process known as missing self recognition (Kärre et al., 1986). Since SHP-1 and SHIP negatively regulate activating receptor signaling, one might predict that the loss of these molecules would boost the effector function of NK cells. Surprisingly, however, SHP-1 and SHIP deficiency in NK cells renders them less functional than their WT counterparts (Lowin-Kropf et al., 2000; Viant et al., 2014; Gumbleton et al., 2015). One explanation of this seemingly paradoxical finding is that NK cells continuously adjust their responsiveness to activating stimuli in their local environment, a phenomenon known as tuning (Höglund and Brodin, 2010). Thus, NK cells that chronically lack inhibitory signals, such as in SHP-1 or SHIP deficiency, require more stimulation to achieve their threshold of activation (Lowin-Kropf et al., 2000; Viant et al., 2014; Gumbleton et al., 2015). While NK cell tuning may protect the host from NK cell-mediated immunopathology, this process can hamper important effector responses against chronic viral infections or tumors.

Although the molecular mechanism of NK cell tuning is unknown, stimulation of NK cells with PMA and a calcium ionophore, ionomycin, can bypass the hyporesponsiveness of SHP-1 and SHIP KO NK cells (Viant et al., 2014; Gumbleton et al., 2015). These data suggest that the tuning process is proximal to PLC $\gamma$-mediated production of DAG. Thus, we speculated that NK cells may not be able to tune their responsiveness in response to enhanced DAG-mediated signaling by $D G K \zeta$ deficiency. Indeed, we recently demonstrated that DGK $\zeta$ KO NK cells are hyperfunctional compared to WT NK cells (Yang et al., 2016). DGK $\zeta$ KO NK cells displayed increased cytokine production and cytotoxicity following stimulation through ITAM, SAP, and DAP10-dependent activating receptors. In contrast, IFN $\gamma$ production by DGK $\zeta$ KO and WT NK cells was similar following stimulation with IL-12 and IL-18, which utilize a DAG-independent signaling pathway, suggesting that the loss of DGK $\zeta$ selectively augmented NK cell responsiveness to DAG-dependent stimuli. Like T cells, the hyperfunctionality of DGK $\zeta$ KO NK cells was dependent on enhanced ERK signaling. Importantly, DGK $\zeta$ KO mice cleared the NK cellsensitive RMA-S tumor more efficiently than WT mice. Thus, the inactivation of negative regulators distal to PLC $\gamma$ such as DGK $\zeta$ might prove therapeutically useful in enhancing NK cell function.

\section{B Cells}

$B$ cells comprise the second arm of the adaptive immune system and are critical for the generation of protective antibody responses during infection. The induction of antibody production results from the stimulation of the somatically rearranged $\mathrm{B}$ cell receptor (BCR) by cognate antigen (McHeyzerWilliams and McHeyzer-Williams, 2005; Kurosaki et al., 2010). Similar to the TCR, activation of the BCR leads to downstream biochemical cascades that ultimately result in the generation of DAG through PLC $\gamma$ and, subsequently, the activation of ERK (Hashimoto et al., 2000; Saijo et al., 2003). ERK has been shown to play multiple roles during B cell responses, including the promotion of B cell survival, proliferation, and differentiation into antibody-secreting plasma cells (Richards et al., 2001; Coughlin et al., 2005; Yasuda et al., 2011). Furthermore, attenuation of ERK activation has been shown to important during B cell development, since ERK signals decrease as $B$ cells progress from the immature transitional stage to mature follicular B cells (Yasuda et al., 2008; Gross et al., 2009; Rowland et al., 2010).

Given the role of ERK in these B cell processes, controlling the levels of BCR-induced DAG through DGK $\zeta$ might be important in regulating $\mathrm{B}$ cell development, activation, and antibody secretion capabilities. For example, mRNA transcripts of DGK $\zeta$ are upregulated as B cells progress from early transitional to the mature follicular stage, which is associated with decreased ERK activation (Wheeler et al., 2013). Accordingly, the loss of DGK $\zeta$ only affected ERK activation and I $\mathrm{B} \alpha$ degradation in the follicular but not early immature transitional B cell pool in response to BCR stimulation. Importantly, the augmentation of 
BCR-induced activation in DGK $\zeta$ KO follicular B cells was seen even under less optimal BCR activation conditions, suggesting that $\mathrm{DGK} \zeta$ might control the BCR activation threshold in these cells.

The effects of DGK $\zeta$ on B cell signaling threshold translate to functional consequences on $\mathrm{B}$ cell effector responses. BCR stimulation of purified DGK $\zeta$ KO splenic B cells in vitro led to increased expression of $\mathrm{CD} 69$ and enhanced proliferation compared to WT B cells. DGK $\zeta$ KO mice displayed enhanced antibody responses to $\mathrm{T}$-independent and $\mathrm{T}$-dependent antigens (Wheeler et al., 2013). The heightened antibody response by DGK $\zeta$-deficiency was accompanied by increased antigenspecific expansion of both germinal center (GC) B cells and plasma cells. These results demonstrate that regulation of DAGdependent ERK activation by DGK $\zeta$ is critical for selectively controlling the activation threshold of mature B cells to limit their activation.

\section{THE IMMUNOMODULATORY ROLE OF DGK $\zeta$}

We have so far described how the loss or inhibition of DGK $\zeta$ can lead to increased immune responses against viruses or cancer. As DGK $\zeta$ is a negative regulator of DAG-mediated signaling, it is conceivable that immune responses would be enhanced in the absence of DGK $\zeta$. However, DGK $\zeta$ deficiency may also lead to dampening or regulation of immune responses. In the sections below, we will discuss how the absence of DGK $\zeta$ can direct and indirectly suppress or modulate rather than enhance immune responses.

\section{Regulatory T Cells}

Regulatory $\mathrm{T}$ cells (Tregs) are a key subset of $\mathrm{T}$ cells that display suppressive function and are important for the regulation of adaptive immune responses. Tregs are governed by the master transcription factor, forkhead box P3 (Foxp3), and exert their immunosuppressive function via the production of immunoregulatory cytokines and through cell contact dependent mechanisms (Josefowicz et al., 2012). Loss of function mutations in the Foxp3 gene, as seen in Scurfy mice and humans with immune dysregulation, polyendocrinopathy, and X-linked lymphoproliferative disease (IPEX), leads to lethal systemic autoimmunity early in life, highlighting the importance of Tregs in inducing immunotolerance against self antigens (Chatila et al., 2000; Bennett et al., 2001; Brunkow et al., 2001; Wildin et al., 2001).

$\mathrm{T}$ cells that strongly recognize self antigens are deleted during thymic development in a process known as negative selection. Specifically, T cells that receive strong TCR signals in the thymus, implying overt self reactivity, undergo apoptosis. As an alternative fate, strong TCR stimulation in developing thymocytes can also lead to Treg differentiation (Josefowicz et al., 2012). Thus, we hypothesized that enhancement of TCRmediated DAG signaling by DGK $\zeta$ deficiency in developing thymocytes may increase Treg generation. Indeed, the loss of DGK $\zeta$ resulted in a significant increase in Treg development in the thymus in a cell-intrinsic manner (Schmidt et al., 2013). DAG-mediated signaling leads to the activation of the NF-кB (through activation of PKC) and ERK pathways. One NF-кB family member, c-Rel, was previously shown to be important for inducing Foxp3 expression in thymocytes (Long et al., 2009; Ruan et al., 2009). Although Treg generation in DGK $\zeta$ KO mice was reduced in the absence of $\mathrm{c}-\mathrm{Rel}$, there was still residual Tregs in the thymus, suggesting that c-Rel was only partially responsible for the increased generation of Tregs in DGK $\zeta$ KO mice (Schmidt et al., 2013). In fact, ERK activation appeared to be more important in the enhancement of Treg generation in DGK $\zeta \mathrm{KO}$ mice. Using an in vitro Treg development assay, we found that the inhibition of ERK phosphorylation by a MEK inhibitor led to decreased Treg generation in a dose-dependent manner, whereby the level of phosphorylated ERK (pERK) directly correlated to the magnitude of Treg generation. Importantly, Treg generation was also increased in sevenmaker mice (Sharp et al., 1997), which express a gain of function ERK mutation that leads to increased resistance to dephosphorylation of active pERK, suggesting that the selective enhancement of the ERK pathway alone is sufficient to increase Treg generation.

In addition to Treg generation in the thymus, TCR signaling plays an important role in the function of Tregs. Although some Treg function may be preserved in the absence of TCR signaling, we demonstrated that Tregs lacking SLP-76 cannot suppress TCR-driven proliferation of conventional T cells (Schmidt et al., 2015). Furthermore, Tregs with a $\mathrm{Y} \rightarrow \mathrm{F}$ mutation at tyrosine 145 (Y145F) of SLP-76, which leads to defective PLC $\gamma$ activation, also display attenuated suppressive function, suggesting that PLC $\gamma$ is important for Treg function. Consistent with this notion, Tregs lacking DGK $\zeta$ display significantly increased suppression of TCR-driven conventional $\mathrm{T}$ cell proliferation compared to WT Tregs. Together, these data demonstrate that DGK $\zeta$ limit Treg generation and function. Thus, DGK $\zeta$ deficiency may indirectly lead to the suppression of immune responses through Tregs.

\section{Mast Cells}

Mast cells are critical mediators in type 2 immune responses involved in protection against helminthes and in pathologic responses in asthma and allergy (Locksley, 2010; Voehringer, 2013). A key feature of mast cell function is the immediate release of pre-formed inflammatory mediators such as histamine, cytokines, and proteases in a process called degranulation. In addition, mast cells produce arachidonic acid metabolites and cytokines in a protracted manner (Voehringer, 2013). One major stimulus for the release of these inflammatory mediators is crosslinking of FceRI, the high affinity receptor for the Fc region of immunoglobulin E (Kinet, 1999).

The interaction of allergens with IgE-Fce RI complexes results in formation of signaling complexes that converge on the activation of PLC $\gamma$ (Atkinson et al., 1992; Schneider et al., 1992). PLC $\gamma$ and subsequent PKC activation have been shown to be critical in controlling mast cell degranulation, suggesting that controlling the levels of DAG might be important for regulating this process (Nechushtan et al., 2000; Wang et al., 2000; Leitges 
et al., 2002; Wen et al., 2002). Indeed, the loss of DGK $\zeta$ in Fce RIstimulated mast cells leads to increased DAG levels, along with enhancement of downstream DAG-dependent signals, including RasGTP and ERK (Olenchock et al., 2006b). Accordingly, DGK $\zeta$ deficiency leads to enhanced mast cell production of IL-6 following FceRI stimulation.

Intriguingly, however, Fc $\varepsilon$ RI-stimulated DGK $\zeta$ KO mast cells display impaired degranulation and are resistant to local skin anaphylaxis (Olenchock et al., 2006b). The differential effect of DGK $\zeta$ deficiency on mast function (the hypersecretion of IL-6 vs. decreased degranulation) may be explained by the negative feedback of DAG on PLC $\gamma$ activation in mast cells. The elevation of DAG by DGK $\zeta$ deficiency appears to negatively regulate the phosphorylation and subsequent activity of PLC $\gamma$. Thus, although DAG accumulates, the production of $\mathrm{IP}_{3}$, and hence $\mathrm{Ca}^{2+}$ flux is attenuated in DGK $\zeta$ KO mast cells. As degranulation responses are highly dependent on elevation of intracellular $\mathrm{Ca}^{2+}$ levels, this may cause a differential effect on degranulation and cytokine production by mast cells (Ozawa et al., 1993; Olenchock et al., 2006b). Thus, as opposed to T cells and NK cells, DGK $\zeta$ exerts both activating and inhibitory effects on mast cell functional responses.

\section{Macrophages and Dendritic Cells}

Macrophages and dendritic cells (DC) play a key role in bridging the adaptive and innate immune responses (Medzhitov, 2001; Janeway and Medzhitov, 2002; Akira and Takeda, 2004). Toll-like receptors (TLR) serve as an important mechanism for equipping macrophages and DCs with the ability to recognize the presence of pathogenic infection and, subsequently, instruct adaptive immune cells on the type of response needed to effectively clear the infection. TLRs can signal through either MyD88 and/or TRIF to induce activation of the NF- $\kappa$ B and ERK pathways (Akira and Takeda, 2004). While TLR activation does not generally lead to PLC $\gamma$ activation, DAG has been shown to be induced in macrophages following stimulation with LPS (TLR4 agonist) and lipopeptide (TLR2 agonist) (Monick et al., 1999; Zhang et al., 2001a,b). Furthermore, inhibition of PLC or PLD reduced cytokine production and nitric oxide formation by macrophages following TLR stimulation, suggesting that control of DAG levels through DGK might be important in regulating TLR-mediated responses.

Interestingly, modulation of DAG levels by the loss of DGK $\zeta$ resulted in impairment rather than enhancement of cytokine production by macrophages and DCs in response to TLR stimulation. Specifically, in a developmentally independent manner, bone marrow derived macrophages (BMM $\Phi)$ and splenic DCs produced substantially less IL-12p40 and TNF $\alpha$ following in vitro stimulation through a variety of TLR agonists (Liu et al., 2007). This paradoxical finding may be explained by the role of DGK in converting DAG into PA. Biochemical analysis revealed that the loss of $\mathrm{DGK} \zeta$ resulted in selective elevation of the PI3K-Akt pathway but no difference in activation of the ERK or NK- $\kappa \mathrm{B}$ pathways following TLR stimulation. Activation of the PI3K pathway has been shown to negatively regulate TLR stimulation (Fukao et al., 2002; Guha and Mackman, 2002; Martin et al., 2005) and chemical inhibition of the
PI3K restored LPS-induced IL-12p40 production from DGK $\zeta$ KO BMMФs (Liu et al., 2007). Intriguingly, the addition of PA also restored LPS-induced IL-12p40 production, suggesting that the cytokine production defect in DGK $\zeta$ KO DCs and macrophages may be due to reduced PA rather than elevated DAG levels. Exactly how PA rescues TLR-induced cytokine production is unknown, but one possible mechanism is through the recruitment of SHP-1 to negatively regulate PI3K activation (Cuevas et al., 1999; Frank et al., 1999; Zhang et al., 2003).

Defective cytokine production was also observed in vivo following intraperitoneal injection of TLR agonists, which correlated with enhanced survival of DGK $\zeta$ KO mice after LPSinduced septic shock (Liu et al., 2007). However, while DGK $\zeta$ KO mice were protected from TLR-mediated pathology, the loss of DGK $\zeta$ conferred susceptibility to Toxoplasma gondii. DGK $\zeta$ KO mice infected with $T$. gondii displayed decreased serum IL$12 \mathrm{p} 40$ and IFN $\gamma$ levels compared to WT mice. Furthermore, IFN $\gamma$ production by $\mathrm{DGK} \zeta \mathrm{KO}$ splenocytes isolated at day 15 and 30 post-infection was significantly attenuated following restimulation with $T$. gondii antigen STAg. Intriguingly, total $\mathrm{CD}^{+}$and $\mathrm{CD}^{+} \mathrm{T}$ cell numbers were similar between WT and DGK $\zeta$ KO mice following infection with the frequency of $\mathrm{CD} 44^{+} \mathrm{CD}^{2} 2^{\text {lo }}$ effector $\mathrm{T}$ cells higher in infected DGK $\zeta \mathrm{KO}$ mice. As TLR-induced IL-12p40 production and the subsequent induction of a Th1 response are critical for protection against T. gondii infection, the impairment of immune responses against $T$. gondii by DGK $\zeta \mathrm{KO}$ mice could be secondary to a defect in macrophage and DC-derived cytokines that drive Th1 responses.

\section{THE ROLE OF OTHER DGK ISOFORMS ON DGK $\zeta$-REGULATED IMMUNE FUNCTION}

So far, we have discussed isoform-specific regulation of immune function by $\mathrm{DGK} \zeta$, however it is possible that the loss of DGK $\zeta$ has other functional consequences that might be masked by redundant functions of other DGK isoforms. Indeed, DGK $\alpha$ has been shown to display some redundant function with DGK $\zeta$ during conventional T and invariant NKT cell (iNKT) development. While singly-deficient DGK $\alpha$ KO and DGK $\zeta$ KO mice display no gross defects in thymic $\mathrm{T}$ cell development, mice deficient in both DGK $\alpha$ and DGK $\zeta$ (DGK $\alpha \zeta$ DKO) have significant reductions in CD4 and CD8 SP populations in the thymus due to a cell-intrinsic block in positive selection from the DP to SP stage (Guo et al., 2008). Interestingly, the addition of PA to fetal thymic organ cultures could partially restore $\mathrm{T}$ cell maturation defect in $\mathrm{DGK} \alpha \zeta$ DKO thymocytes, suggesting that DGK $\alpha$ and $\mathrm{DGK} \zeta$ regulate $\mathrm{T}$ cell development partly through redundant production of PA.

Similarly, the development of iNKT cells is intact in mice singly-deficient for either DGK $\alpha$ or DGK $\zeta$ (Shen et al., 2011). However, the loss of both DGK $\alpha$ and DGK $\zeta$ results in a complete impairment of iNKT cell maturation in the thymus, spleen, and liver at both early and terminal stages in a cell-intrinsic 
manner. Selective enhancement of the ERK pathway through the expression of a constitutively active K-ras resulted in a significant reduction in mature iNKT cells due a block in Stage II to Stage III maturation of iNKT precursors. Furthermore, augmented activation of the NF- $\kappa \mathrm{B}$ pathway through the expression of a constitutively active IKK $\beta$ also resulted in an impairment in iNKT maturation at both early and terminal stages of development. These results suggest that DGK $\alpha$ and DGK $\zeta$ play redundant roles in the regulation of iNKT maturation by controlling DAG-mediated activation of the ERK and NF- $\kappa$ B pathways.

In addition to controlling innate and conventional $\mathrm{T}$ cell development, $\mathrm{DGK} \alpha$ has also been shown to promote $\mathrm{T}$ cell anergy in conjunction with DGK $\zeta$. Overexpression of either $\mathrm{DGK} \alpha$ or DGK $\zeta$ in Jurkat $\mathrm{T}$ cells induces an anergic-like state that is highlighted by reduced DAG-dependent TCR signals without the impairment of calcium flux (Olenchock et al., 2006a). Similar to DGK $\zeta$ KO T cells, $\mathrm{T}$ cells deficient in $\mathrm{DGK} \alpha$ resist anergy induction when activated through their TCR in the absence of costimulation and during superantigeninduced activation. Furthermore, pharmacological inhibition of DGK $\alpha$ in DGK $\zeta$-deficient $\mathrm{T}$ cells can further enhance proliferation and IL-2 production in response to anergy-inducing conditions, suggesting that both DGK $\alpha$ and DGK $\zeta$ contribute to anergy induction in inappropriately activated $\mathrm{T}$ cells through the synergistic regulation of TCR-induced DAG-mediated signaling.
While DGK $\alpha$ and DGK $\zeta$ can share similar functions, DGK $\alpha$ does not simply compensate for all DGK $\zeta$-regulated functions. For example, unlike DGK $\zeta$ KO mice, DGK $\alpha$-deficient mice do not display an enhancement in Treg generation in the thymus or hyperfunctional NK cell responses, thus emphasizing that the regulation of these processes by DGKs is isoform-specific and unique to DGK $\zeta$ (Joshi et al., 2013; Yang et al., 2016). The independent and redundant roles of DGK $\alpha$ and other DGK isoforms on DGK $\zeta$-regulated functions in other immune cells remain unexplored.

\section{CONCLUDING REMARKS}

As a negative regulator of DAG-mediated signaling, one might predict that the loss of DGK $\zeta$ would universally lead to immune activation. Interestingly, however, the inhibition of DGK $\zeta$ does not only enhance but also suppresses selective immune responses (Figure 1). Thus, although DGK $\zeta$ may represent a drug target for enhancing cytotoxic $\mathrm{T}$ cell responses against cancer, it may also simultaneously serve as a target for the treatment of allergic responses or septic shock. In addition to activation or inhibition of immune responses, DGK $\zeta$ may also play a role in modulating the immune response. For example, it has been shown that the differentiation of $\mathrm{CD} 4{ }^{+} \mathrm{T}$ cells into Th1 and Th2 subsets may be dependent on TCR signal strength, whereby strong TCR-induced ERK signals favor Th1 over Th2 differentiation (Yamane et al.,

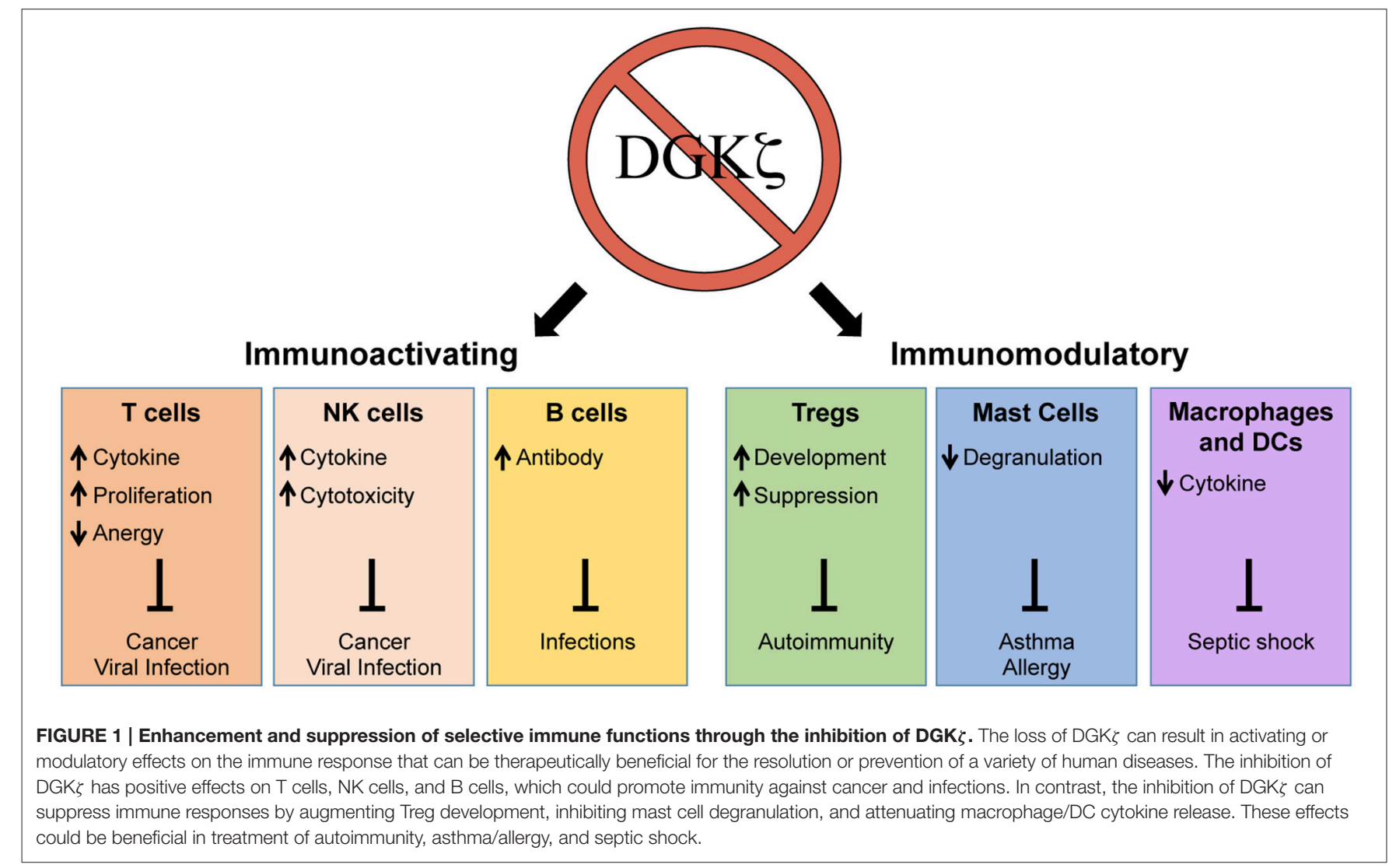


2005). Thus, it is possible that DGK $\zeta$ controls Th1 vs. Th2 lineage commitment by regulating DAG-mediated ERK activation in T cells. Although the impact of DGK $\zeta$ deficiency on Th2 responses is unknown, DGK $\zeta$ KO T cells display heightened Th1-driven anti-viral and anti-tumor responses. Thus, it would be interesting to test whether the loss of DGK $\zeta$ impairs protection against Th2inducing helminth infections or beneficially promotes protection against Th2-mediated diseases such as asthma. The precise delineation of how DGK $\zeta$ controls the outcome of immune responses will yield insight into how DGK $\zeta$ could be targeted for the treatment of various immune-mediated disorders.

\section{REFERENCES}

Akira, S., and Takeda, K. (2004). Toll-like receptor signalling. Nat. Rev. Immunol. 4, 499-511. doi: 10.1038/nri1391

Artis, D., and Spits, H. (2015). The biology of innate lymphoid cells. Nature 517, 293-301. doi: 10.1038/nature14189

Atkinson, T. P., Kaliner, M. A., and Hohman, R. J. (1992). Phospholipase C-gamma 1 is translocated to the membrane of rat basophilic leukemia cells in response to aggregation of IgE receptors. J. Immunol. 148, 2194-2200.

Bennett, C. L., Christie, J., Ramsdell, F., Brunkow, M. E., Ferguson, P. J., Whitesell, L., et al. (2001). The immune dysregulation, polyendocrinopathy, enteropathy, X-linked syndrome (IPEX) is caused by mutations of FOXP3. Nat. Genet. 27, 20-21. doi: 10.1038/83713

Binstadt, B. A., Brumbaugh, K. M., Dick, C. J., Scharenberg, A. M., Williams, B. L., Colonna, M., et al. (1996). Sequential involvement of Lck and SHP-1 with MHC-recognizing receptors on NK Cells inhibits FcR-initiated tyrosine kinase activation. Immunity 5, 629-638. doi: 10.1016/S1074-7613(00)80276-9

Brunkow, M. E., Jeffery, E. W., Hjerrild, K. A., Paeper, B., Clark, L. B., Yasayko, S.-A., et al. (2001). Disruption of a new forkhead/winged-helix protein, scurfin, results in the fatal lymphoproliferative disorder of the scurfy mouse. Nat. Genet. 27, 68-73. doi: 10.1038/83784

Bunting, M., Tang, W., Zimmerman, G. A., McIntyre, T. M., and Prescott, S. M. (1996). Molecular cloning and characterization of a novel human diacylglycerol kinase zeta. J. Biol. Chem. 271, 10230-10236. doi: 10.1074/jbc.271.17.10237

Chatila, T. A., Blaeser, F., Ho, N., Lederman, H. M., Voulgaropoulos, C., Helms, C., et al. (2000). JM2, encoding a fork head-related protein, is mutated in X-linked autoimmunity-allergic disregulation syndrome. J. Clin. Invest. 106, R75-R81. doi: 10.1172/JCI11679

Chen, R., Latour, S., Shi, X., and Veillette, A. (2006). Association between SAP and FynT: inducible SH3 domain-mediated interaction controlled by engagement of the SLAM receptor. Mol. Cell. Biol. 26, 5559-5568. doi: 10.1128/MCB.00357-06

Coudronniere, N., Villalba, M., Englund, N., and Altman, A. (2000). NF$\kappa \mathrm{B}$ activation induced by $\mathrm{T}$ cell receptor/CD28 costimulation is mediated by protein kinase C- $\theta$. Proc. Natl. Acad. Sci. U.S.A. 97, 3394-3399. doi: 10.1073/pnas.060028097

Coughlin, J. J., Stang, S. L., Dower, N. A., and Stone, J. C. (2005). RasGRP1 and RasGRP3 regulate $B$ cell proliferation by facilitating B cell receptor-Ras signaling. J. Immunol. 175, 7179-7184. doi: 10.4049/jimmunol.175.11.7179

Cuevas, B., Lu, Y., Watt, S., Kumar, R., Zhang, J., Siminovitch, K. A., et al. (1999). SHP-1 regulates Lck-induced phosphatidylinositol 3-kinase phosphorylation and activity. J. Biol. Chem. 274, 27583-27589. doi: 10.1074/jbc.274.39.27583

Dower, N. A., Stang, S. L., Bottorff, D. A., Ebinu, J. O., Dickie, P., Ostergaard, H. L., et al. (2000). RasGRP is essential for mouse thymocyte differentiation and TCR signaling. Nat. Immunol. 1, 317-321. doi: 10.1038/79766

Frank, C., Keilhack, H., Opitz, F., Zschörnig, O., and Böhmer, F. D. (1999). Binding of phosphatidic acid to the protein-tyrosine phosphatase SHP-1 as a basis for activity modulation. Biochemistry 38, 11993-12002. doi: 10.1021/bi98 $2586 \mathrm{w}$

Fukao, T., Tanabe, M., Terauchi, Y., Ota, T., Matsuda, S., Asano, T., et al. (2002). PI3K-mediated negative feedback regulation of IL-12 production in DCs. Nat. Immunol. 3, 875-881. doi: 10.1038/ni825

\section{AUTHOR CONTRIBUTIONS}

All authors listed, have made substantial, direct and intellectual contribution to the work, and approved it for publication.

\section{ACKNOWLEDGMENTS}

This work was supported by grants from the Translational Center of Excellence in Hematological Malignancies of the Abramson Cancer Center and by National Institutes of Health Grants R01HL107589 and R01HL111501.

Goto, K., and Kondo, H. (1996). A 104-kDa diacylglycerol kinase containing ankyrin-like repeats localizes in the cell nucleus. Proc. Natl. Acad. Sci. U.S.A. 93, 11196-11201. doi: 10.1073/pnas.93.20.11196

Gross, A. J., Lyandres, J. R., Panigrahi, A. K., Prak, E. T. L., and DeFranco, A. L. (2009). Developmental acquisition of the Lyn-CD22-SHP-1 inhibitory pathway promotes B cell tolerance. J. Immunol. 182, 5382-5392. doi: 10.4049/jimmunol.0803941

Guha, M., and Mackman, N. (2002). The phosphatidylinositol 3-kinase-Akt pathway limits lipopolysaccharide activation of signaling pathways and expression of inflammatory mediators in human monocytic cells. J. Biol. Chem. 277, 32124-32132. doi: 10.1074/jbc.M203298200

Gumbleton, M., Vivier, E., and Kerr, W. G. (2015). SHIP1 intrinsically regulates NK cell signaling and education, resulting in tolerance of an MHC class Imismatched bone marrow graft in mice. J. Immunol. 194, 2847-2854. doi: 10.4049/jimmunol.1402930

Guo, R., Wan, C. K., Carpenter, J. H., Mousallem, T., Boustany, R. M., Kuan, C. T., et al. (2008). Synergistic control of T cell development and tumor suppression by diacylglycerol kinase alpha and zeta. Proc. Natl. Acad. Sci. U.S.A. 105, 11909-11914. doi: 10.1073/pnas.0711856105

Hashimoto, A., Takeda, K., Inaba, M., Sekimata, M., Kaisho, T., Ikehara, S., et al. (2000). Cutting edge: essential role of phospholipase C- $\gamma 2$ in B cell development and function. J. Immunol. 165, 1738-1742. doi: 10.4049/jimmunol.165.4.1738

Höglund, P., and Brodin, P. (2010). Current perspectives of natural killer cell education by MHC class I molecules. Nat. Rev. Immunol. 10, 724-734. doi: $10.1038 /$ nri2835

Imboden, J. B., and Stobo, J. D. (1985). Transmembrane signalling by the T cell antigen receptor. Perturbation of the T3-antigen receptor complex generates inositol phosphates and releases calcium ions from intracellular stores. J. Exp. Med. 161, 446-456.

Janeway, C. A. Jr., and Medzhitov, R. (2002). Innate immune recognition. Annu. Rev. Immunol. 20, 197-216. doi: 10.1146/annurev.immunol.20.083001.084359

Josefowicz, S. Z., Lu, L. F., and Rudensky, A. Y. (2012). Regulatory T cells: mechanisms of differentiation and function. Annu. Rev. Immunol. 30, 531-564. doi: 10.1146/annurev.immunol.25.022106.141623

Joshi, R. P., and Koretzky, G. A. (2013). Diacylglycerol kinases: regulated controllers of T cell activation, function, and development. Int. J. Mol. Sci. 14, 6649-6673. doi: 10.3390/ijms14046649

Joshi, R. P., Schmidt, A. M., Das, J., Pytel, D., Riese, M. J., Lester, M., et al. (2013). The $\zeta$ isoform of diacylglycerol kinase plays a predominant role in regulatory T cell development and TCR-mediated ras signaling. Sci. Signal. 6:ra102. doi: 10.1126/scisignal.2004373

Jung, H., Hsiung, B., Pestal, K., Procyk, E., and Raulet, D. H. (2012). RAE-1 ligands for the NKG2D receptor are regulated by E2F transcription factors, which control cell cycle entry. J. Exp. Med. 209, 2409-2422. doi: 10.1084/jem.20120565

Kambayashi, T., and Koretzky, G. A. (2007). Proximal signaling events in Fc epsilon RI-mediated mast cell activation. J. Allergy Clin. Immunol. 119, 544-552; quiz 553-544. doi: 10.1016/j.jaci.2007.01.017

Kärre, K., Ljunggren, H. G., Piontek, G., and Kiessling, R. (1986). Selective rejection of $\mathrm{H}$-2-deficient lymphoma variants suggests alternative immune defence strategy. Nature 319, 675-678. 
Kinet, J. P. (1999). The high-affinity IgE receptor (FceRI): from physiology to pathology. Annu. Rev. Immunol. 17, 931-972. doi: 10.1146/annurev.immunol. 17.1.931

Kobayashi, N., Hozumi, Y., Ito, T., Hosoya, T., Kondo, H., and Goto, K. (2007). Differential subcellular targeting and activity-dependent subcellular localization of diacylglycerol kinase isozymes in transfected cells. Eur. J. Cell Biol. 86, 433-444. doi: 10.1016/j.ejcb.2007.05.002

Kurosaki, T., Shinohara, H., and Baba, Y. (2010). B cell signaling and fate decision. Annu. Rev. Immunol. 28, 21-55. doi: 10.1146/annurev.immunol. 021908.132541

Lanier, L. L. (2008). Up on the tightrope: natural killer cell activation and inhibition. Nat. Immunol. 9, 495-502. doi: 10.1038/ni1581

Leitges, M., Gimborn, K., Elis, W., Kalesnikoff, J., Hughes, M. R., Krystal, G., et al. (2002). Protein Kinase C- Is a negative regulator of antigen-induced mast cell degranulation. Mol. Cell. Biol. 22, 3970-3980. doi: 10.1128/MCB.22.12.39703980.2002

Liu, C.-H. H., Machado, F. S., Guo, R., Nichols, K. E., Burks, A. W., Aliberti, J. C., et al. (2007). Diacylglycerol kinase zeta regulates microbial recognition and host resistance to Toxoplasma gondii. J. Exp. Med. 204, 781-792. doi: 10.1084/jem.20061856

Locksley, R. M. (2010). Asthma and allergic inflammation. Cell 140, 777-783. doi: 10.1016/j.cell.2010.03.004

Long, M., Park, S. G., Strickland, I., Hayden, M. S., and Ghosh, S. (2009). Nuclear factor- $\kappa \mathrm{B}$ modulates regulatory $\mathrm{T}$ cell development by directly regulating expression of Foxp3 transcription factor. Immunity 31, 921-931. doi: 10.1016/j.immuni.2009.09.022

Lowin-Kropf, B., Kunz, B., Beermann, F., and Held, W. (2000). Impaired natural killing of MHC class I-deficient targets by NK cells expressing a catalytically inactive form of SHP-1. J. Immunol. 165, 1314-1321. doi: 10.4049/jimmunol.165.3.1314

Macian, F., Garcia-Cozar, F., Im, S. H., Horton, H. F., Byrne, M. C., and Rao, A. (2002). Transcriptional mechanisms underlying lymphocyte tolerance. Cell 109, 719-731. doi: 10.1016/S0092-8674(02)00767-5

Martin, M., Rehani, K., Jope, R. S., and Michalek, S. M. (2005). Toll-like receptormediated cytokine production is differentially regulated by glycogen synthase kinase 3. Nat. Immunol. 6, 777-784. doi: 10.1038/ni1221

May, R. M., Okumura, M., Hsu, C. J., Bassiri, H., Yang, E., Rak, G., et al. (2013). Murine natural killer immunoreceptors use distinct proximal signaling complexes to direct cell function. Blood 121, 3135-3146. doi: 10.1182/blood2012-12-474361

McHeyzer-Williams, L. J., and McHeyzer-Williams, M. G. (2005). Antigenspecific memory B cell development. Annu. Rev. Immunol. 23, 487-513. doi: 10.1146/annurev.immunol.23.021704.115732

McVicar, D. W., Taylor, L. S., Gosselin, P., Willette-Brown, J., Mikhael, A. I., Geahlen, R. L., et al. (1998). DAP12-mediated signal transduction in natural killer cells. A dominant role for the Syk protein-tyrosine kinase. J. Biol. Chem. 273, 32934-32942.

Medzhitov, R. (2001). Toll-like receptors and innate immunity. Nat. Rev. Immunol. 1, 135-145. doi: 10.1038/35100529

Monick, M. M., Carter, A. B., Gudmundsson, G., Mallampalli, R., Powers, L. S., and Hunninghake, G. W. (1999). A phosphatidylcholine-specific phospholipase $\mathrm{C}$ regulates activation of $\mathrm{p} 42 / 44$ mitogen-activated protein kinases in lipopolysaccharide-stimulated human alveolar macrophages. J. Immunol. 162, 3005-3012.

Nechushtan, H., Leitges, M., Cohen, C., Kay, G., and Razin, E. (2000). Inhibition of degranulation and interleukin-6 production in mast cells derived from mice deficient in protein kinase C $\beta$. Blood 95, 1752.

Olenchock, B. A., Guo, R., Carpenter, J. H., Jordan, M., Topham, M. K., Koretzky, G. A., et al. (2006a). Disruption of diacylglycerol metabolism impairs the induction of T cell anergy. Nat. Immunol. 7, 1174-1181. doi: 10.1038/nil400

Olenchock, B. A., Guo, R., Silverman, M. A., Wu, J. N., Carpenter, J. H., Koretzky, G. A., et al. (2006b). Impaired degranulation but enhanced cytokine production after Fc epsilonRI stimulation of diacylglycerol kinase zeta-deficient mast cells. J. Exp. Med. 203, 1471-1480. doi: 10.1084/jem.20052424

Ozawa, K., Szallasi, Z., Kazanietz, M. G., Blumberg, P. M., Mischak, H., Mushinski, J. F., et al. (1993). Ca(2+)-dependent and $\mathrm{Ca}(2+)$-independent isozymes of protein kinase $\mathrm{C}$ mediate exocytosis in antigen-stimulated rat basophilic RBL$2 \mathrm{H} 3$ cells. Reconstitution of secretory responses with $\mathrm{Ca} 2+$ and purified isozymes in washed permeabilized cells. J. Biol. Chem. 268, 1749-1756.

Quann, E. J., Liu, X., Altan-Bonnet, G., and Huse, M. (2011). A cascade of protein kinase $\mathrm{C}$ isozymes promotes cytoskeletal polarization in T cells. Nat. Immunol. 12, 647-654. doi: 10.1038/ni.2033

Richards, J. D., Dave, S. H., Chou, C. H., Mamchak, A. A., and DeFranco, A. L. (2001). Inhibition of the MEK/ERK signaling pathway blocks a subset of B cell responses to antigen. J. Immunol. 166, 3855-3864. doi: 10.4049/jimmunol.166.6.3855

Riese, M. J., Grewal, J., Das, J., Zou, T., Patil, V., Chakraborty, A. K., et al. (2011). Decreased diacylglycerol metabolism enhances ERK activation and augments CD8+ T cell functional responses. J. Biol. Chem. 286, 5254-5265. doi: 10.1074/jbc.M110.171884

Riese, M. J., Wang, L.-C. S. C., Moon, E. K., Joshi, R. P., Ranganathan, A., June, C. H., et al. (2013). Enhanced effector responses in activated CD8+ $\mathrm{T}$ cells deficient in diacylglycerol kinases. Cancer Res. 73, 3566-3577. doi: 10.1158/0008-5472.CAN-12-3874

Rowland, S. L., DePersis, C. L., Torres, R. M., and Pelanda, R. (2010). Ras activation of Erk restores impaired tonic BCR signaling and rescues immature B cell differentiation. J. Exp. Med. 207, 607-621. doi: 10.1084/jem.20091673

Ruan, Q., Kameswaran, V., Tone, Y., Li, L., Liou, H. C., Greene, M. I., et al. (2009). Development of Foxp3(+) regulatory $t$ cells is driven by the c-Rel enhanceosome. Immunity 31, 932-940. doi: 10.1016/j.immuni.2009.10.006

Rui, T., Zhang, J., Xu, X., Yao, Y., Kao, R., and Martin, C. M. (2012). Reduction in IL-33 expression exaggerates ischaemia/reperfusion-induced myocardial injury in mice with diabetes mellitus. Cardiovasc. Res. 94, 370-378. doi: $10.1093 /$ cvr/cvs015

Saijo, K., Schmedt, C., Su, I. H. H., Karasuyama, H., Lowell, C. A., Reth, M., et al. (2003). Essential role of Src-family protein tyrosine kinases in NF- $\kappa$ B activation during B cell development. Nat. Immunol. 4, 274-279. doi: 10.1038/ni893

Schmidt, A. M., Lu, W., Sindhava, V. J., Huang, Y., Burkhardt, J. K., Yang, E., et al. (2015). Regulatory T cells require TCR signaling for their suppressive function. J. Immunol. 194, 4362-4370. doi: 10.4049/jimmunol.1402384

Schmidt, A. M., Zou, T., Joshi, R. P., Leichner, T. M., Pimentel, M. A., Sommers, C. L., et al. (2013). Diacylglycerol kinase $\zeta$ limits the generation of natural regulatory T cells. Sci. Signal. 6:ra101. doi: 10.1126/scisignal.2004411

Schneider, H., Cohen-Dayag, A., and Pecht, I. (1992). Tyrosine phosphorylation of phospholipase $C(\gamma 1)$ couples the $F c \varepsilon$ receptor mediated signal to mast cells secretion. Int. Immunol. 4, 447-453. doi: 10.1093/intimm/4.4.447

Sharp, L. L., Schwarz, D. A., Bott, C. M., Marshall, C. J., and Hedrick, S. M. (1997). The influence of the MAPK pathway on T cell lineage commitment. Immunity 7, 609-618. doi: 10.1016/S1074-7613(00)80382-9

Shen, S., Wu, J., Srivatsan, S., Gorentla, B. K., Shin, J., Xu, L., et al. (2011). Tight regulation of diacylglycerol-mediated signaling is critical for proper invariant NKT cell development. J. Immunol. 187, 2122-2129. doi: 10.4049/jimmunol.1100495

Shulga, Y. V., Topham, M. K., and Epand, R. M. (2011). Regulation and functions of diacylglycerol kinases. Chem. Rev. 111, 6186-6208. doi: 10.1021/cr1004106

Smith-Garvin, J. E., Koretzky, G. A., and Jordan, M. S. (2009). T cell activation. Annu. Rev. Immunol. 27, 591-619. doi: 10.1146/annurev. immunol.021908.132706

Sun, Z., Arendt, C. W., Ellmeier, W., Schaeffer, E. M., Sunshine, M. J., Gandhi, L., et al. (2000). PKC- $[\theta]$ is required for TCR-induced NF- $[\kappa] B$ activation in mature but not immature $\mathrm{T}$ lymphocytes. Nature 404, 402-407. doi: $10.1038 / 35006090$

Tassi, I., Presti, R., Kim, S., Yokoyama, W. M., Gilfillan, S., and Colonna, M. (2005). Phospholipase $\mathrm{C}-\gamma 2$ is a critical signaling mediator for murine NK cell activating receptors. J. Immunol. 175, 749-754. doi: 10.4049/jimmunol.175.2.749

Tognon, C. E., Kirk, H. E., Passmore, L. A., Whitehead, I. P., Der, C. J., and Kay, R. J. (1998). Regulation of RasGRP via a phorbol ester-responsive C1 domain. Mol. Cell. Biol. 18, 6995-7008. doi: 10.1128/MCB.18.12.6995

Topham, M. K., and Prescott, S. M. (1999). Mammalian diacylglycerol kinases, a family of lipid kinases with signaling functions. J. Biol. Chem. 274, 11447-11450. doi: 10.1074/jbc.274.17.11447 
Viant, C., Fenis, A., Chicanne, G., Payrastre, B., Ugolini, S., and Vivier, E. (2014). SHP-1-mediated inhibitory signals promote responsiveness and anti-tumour functions of natural killer cells. Nat. Commun. 5, 5108. doi: $10.1038 /$ ncomms6108

Voehringer, D. (2013). Protective and pathological roles of mast cells and basophils. Nat. Rev. Immunol. 13, 362-375. doi: 10.1038/nri3427

Wang, D., Feng, J., Wen, R., Marine, J. C., Sangster, M. Y., Parganas, E., et al. (2000). Phospholipase $C \gamma 2$ is essential in the functions of B cell and several Fc receptors. Immunity 13, 25-35. doi: 10.1016/S1074-7613(00)00005-4

Wang, D., Matsumoto, R., You, Y., Che, T., Lin, X. Y., Gaffen, S. L., et al. (2004). CD3/CD28 costimulation-induced NF- $\mathrm{KB}$ activation is mediated by recruitment of protein kinase $C-\theta, B c l 10$, and IкB kinase $\beta$ to the immunological synapse through CARMA1. Mol. Cell. Biol. 24, 164-171. doi: 10.1128/MCB.24.1.164-171.2003

Wen, R., Jou, S.-T. T., Chen, Y., Hoffmeyer, A., and Wang, D. (2002). Phospholipase C- $\gamma 2$ is essential for specific functions of Fc $\varepsilon$ R and Fc $\gamma$ R. J. Immunol. 169, 6743-6752. doi: 10.4049/jimmunol.169.12.6743

Wheeler, M. L., Dong, M. B., Brink, R., Zhong, X. -P. P., and DeFranco, A. L. (2013). Diacylglycerol kinase $\zeta$ limits B cell antigen receptor-dependent activation of ERK signaling to inhibit early antibody responses. Sci. Signal. 6:ra91. doi: 10.1126/scisignal.2004189

Wildin, R. S., Ramsdell, F., Peake, J., Faravelli, F., Casanova, J. L., Buist, N., et al. (2001). X-linked neonatal diabetes mellitus, enteropathy and endocrinopathy syndrome is the human equivalent of mouse scurfy. Nat. Genet. 27, 18-20. doi: $10.1038 / 83707$

Wright, D. B., Tripathi, S., Sikarwar, A., Santosh, K. T., Perez-Zoghbi, J., Ojo, O. O., et al. (2013). Regulation of GPCR-mediated smooth muscle contraction: implications for asthma and pulmonary hypertension. Pulm. Pharmacol. Ther. 26, 121-131. doi: 10.1016/j.pupt.2012.06.005

Wu, J. N., and Koretzky, G. A. (2004). The SLP-76 family of adapter proteins. Semin. Immunol. 16, 379-393. doi: 10.1016/j.smim.2004.08.018

Wu, J., Song, Y., Bakker, A. B., Bauer, S., Spies, T., Lanier, L. L., et al. (1999). An activating immunoreceptor complex formed by NKG2D and DAP10. Science 285, 730-732. doi: 10.1126/science.285.5428.730

Yamane, H., Zhu, J., and Paul, W. E. (2005). Independent roles for IL-2 and GATA3 in stimulating naive CD4+ T cells to generate a Th2-inducing cytokine environment. J. Exp. Med. 202, 793-804. doi: 10.1084/jem.20051304

Yang, E., Singh, B. K., Paustian, A. M., and Kambayashi, T. (2016). Diacylglycerol Kinase $\zeta$ Is a Target To Enhance NK Cell Function. J. Immunol. 197, 934-941. doi: 10.4049/jimmunol.1600581
Yasuda, T., Kometani, K., Takahashi, N., Imai, Y., Aiba, Y., and Kurosaki, T. (2011). ERKs induce expression of the transcriptional repressor Blimp1 and subsequent plasma cell differentiation. Sci. Signal. 4, ra25. doi: 10.1126/scisignal.2001592

Yasuda, T., Sanjo, H., Pagès, G., Kawano, Y., Karasuyama, H., Pouysségur, J., et al. (2008). Erk kinases link pre-B cell receptor signaling to transcriptional events required for early B cell expansion. Immunity 28, 499-508. doi: 10.1016/j.immuni.2008.02.015

Zha, Y., Marks, R., Ho, A. W., Peterson, A. C., Janardhan, S., Brown, I., et al. (2006). T cell anergy is reversed by active Ras and is regulated by diacylglycerol kinase- $\alpha$. Nat. Immunol. 7, 1166-1173. doi: 10.1038/ni1394

Zhang, F., Zhao, G., and Dong, Z. (2001a). Phosphatidylcholine-specific phospholipase C and D in stimulation of RAW264.7 mouse macrophage-like cells by lipopolysaccharide. Int. Immunopharmacol. 1, 1375-1384.

Zhang, F., Zhao, G., and Dong, Z. (2001b). Phosphatidylcholine-specific phospholipase C regulates activation of RAW264.7 macrophage-like cells by lipopeptide JBT3002. J. Leukoc. Biol. 69, 1060-1066.

Zhang, Z., Jimi, E., and Bothwell, A. L. M. (2003). Receptor activator of NF- $\kappa$ B ligand stimulates recruitment of SHP-1 to the complex containing TNFR-associated factor 6 that regulates osteoclastogenesis. J. Immunol. 171, 3620-3626. doi: 10.4049/jimmunol.171.7.3620

Zhong, X.-P. P., Hainey, E. A., Olenchock, B. A., Jordan, M. S., Maltzman, J. S., Nichols, K. E., et al. (2003). Enhanced T cell responses due to diacylglycerol kinase zeta deficiency. Nat. Immunol. 4, 882-890. doi: 10.1038/ ni958

Zhong, X.-P. P., Hainey, E. A., Olenchock, B. A., Zhao, H., Topham, M. K., and Koretzky, G. A. (2002). Regulation of T cell receptor-induced activation of the Ras-ERK pathway by diacylglycerol kinase zeta. J. Biol. Chem. 277, 31089-31098. doi: 10.1074/jbc.M203818200

Conflict of Interest Statement: The authors declare that the research was conducted in the absence of any commercial or financial relationships that could be construed as a potential conflict of interest.

Copyright (c) 2016 Singh and Kambayashi. This is an open-access article distributed under the terms of the Creative Commons Attribution License (CC BY). The use, distribution or reproduction in other forums is permitted, provided the original author(s) or licensor are credited and that the original publication in this journal is cited, in accordance with accepted academic practice. No use, distribution or reproduction is permitted which does not comply with these terms. 